\title{
Failure Analysis on Conveyor Chain Links of a Central Bucket Elevator
}

\author{
Edward Yin ${ }^{1}$, Onumus Muvengei ${ }^{2}$, John Kihiu ${ }^{3}$, Kenneth Njoroge ${ }^{4}$ \\ ${ }^{1,2,3,4}$ Department of Mechanical Engineering, Jomo Kenyatta University of Agriculture and Technology, Kenya)
}

\begin{abstract}
Failure of chain links of a bucket elevator is inevitable. For this reason, analysis of failure is very crucial to the continuous improvement on the chain links which as a result will minimize down-time therefore increasing productivity. Whenever the chain link of the bucket elevator fail, it takes the technicians a minimum of three days depending on the severity of damage. This results in increased down-time which reduces production time coupled with damaged buckets and links which increases maintenance cost. The objective of this research was to analysis the cause of failure of the chain link of a bucket elevator by carrying out visual examination, chemical analysis and metallurgical analysis on failed chain link samples. Preliminary examination using stereo microscope revealed the type of fracture is a brittle fracture and chevron marks showed that the fracture began from the core of the fractured surface and progressed through the material until eventual fracture occurred. Metallurgical analysis revealed that the micro-structure is a tempered martensite and the material had a lot of cracks embedded within it which propagated during loading. Chemical analysis revealed that the carbon content was below the required standards and therefore does not meet the requirements. It was concluded that the crack had initiated from an inclusion and therefore was the cause of failure.
\end{abstract}

Keywords: Bucket Elevator, Chain link, Inclusions, Failure Analysis, Fracture

\section{Introduction}

Central chain bucket elevators are basically designed to move granular materials vertically through a height. The elevators use an endless chain with a series of buckets attached to it. The bulk material is spread into an inlet hopper where the buckets dig into the material, convey it up over the head sprocket. Thereafter, the buckets throw the material out via a discharge spout. The buckets are returned down to the tail sprocket at the bottom.

The bucket elevator consists of buckets to contain the material, chain or belt drive to carry the buckets and transmit the pull. In addition, it also has electrical motor to drive the chain or belt, loading and discharge spout for loading the buckets at the boot and receiving the discharged material at the head respectively. Tensioning device is also installed for maintaining the chain/belt tension. All these accessories are enclosed and protected in a casing [1]. Fig 1 shows the various parts of the bucket elevator.

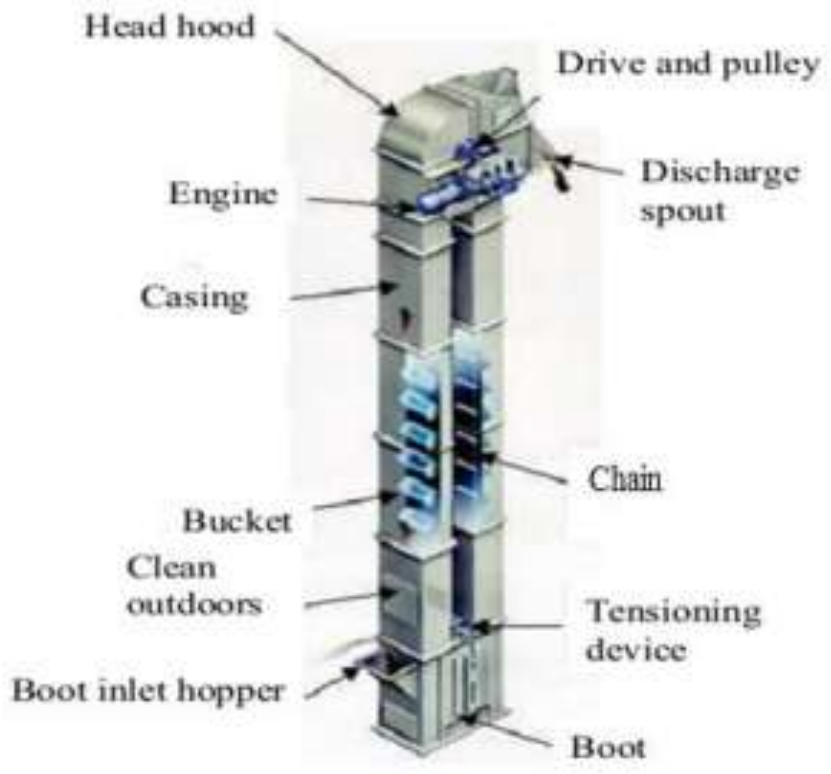

Figure 1: Parts of a bucket elevator 
There are mainly two types of bucket elevators namely; the belt and chain bucket elevator. The main difference between the two is that the former uses an endless belt with pulley whilst the later uses an endless chain with sprocket as shown in Fig 2 (a) and (b).

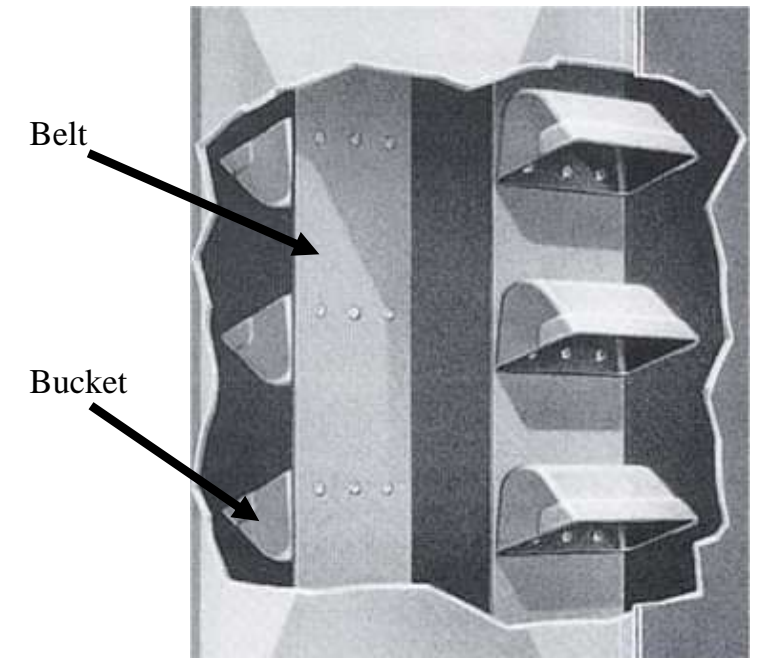

(a)

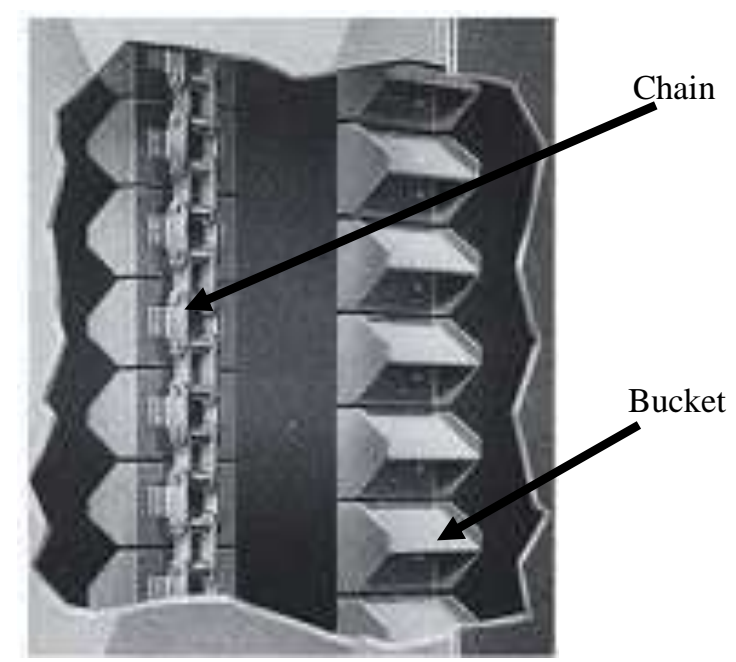

(b)

Figure 2: Belt and Chain bucket elevator

The buckets are designed to stay upright after scooping material to prevent spillage. The head is one of the major structural elements of the overall elevator. It supports the weight of buckets and belt and also accommodates the drive and anti-run back device [2]. Bucket elevators are used in the lime and cement industry for conveying powders, fertilizers, minerals and many others.

Bucket elevators are very important to the extent that the whole production process cease whenever the elevator is not in operation condition and this is mainly caused by failure of chain links. Although other parts such as bearing, shaft, and gears can also fail, they do not occur frequently as compared to chain links. Failure of chain links does not only reduce productivity but also cost of maintenance because of damaged buckets and links associated with the failure.

The bucket elevator investigated in this study has specifications shown in Table 1

Table 1 Specification of bucket elevator

\begin{tabular}{|l|l|}
\hline Item & Quantity \\
\hline Capacity & $80 \mathrm{t} / \mathrm{hr}$ \\
\hline Power Rating & $75 \mathrm{kw}$ \\
\hline Speed & $1.62 \mathrm{~m} / \mathrm{s}$ \\
\hline $\begin{array}{l}\text { Weight of chain link per } \\
\text { unit length }\end{array}$ & $57 \mathrm{~kg} / \mathrm{m}$ \\
\hline Height & $30 \mathrm{~m}$ \\
\hline Width & $2.35 \mathrm{~m}$ \\
\hline Average Temp. & $75^{\circ} \mathrm{C}$ \\
\hline
\end{tabular}

The above data was obtained from East African Portland Cement Company (EAPC), Kenya. On the average the down-town is three (3) days which in terms of hours is $72 \mathrm{hrs}$. Therefore the tons of cement lost during the down-time is 5760 tons $(5,760,000 \mathrm{~kg})$ which in terms of bags of cement is $115,200 \mathrm{bags}$.

It is therefore imperative to analyze the cause of failure so as to improve on the quality of chain links produced which in turn will reduce down-time thereby improving productivity. Down-time is the number of hours for which the bucket elevator is not in operation.

Momcilovic et. al [3] investigated a failed bracket of a conveyor using Scanning Electron Microscopy (SEM) analysis and found significant presence of oxide on the crack surface. The authors noticed that the contact zone between chain link and bracket is one of the most stressed zones and fracture always occurred in that zone. Based on their research, they concluded that the origin of cracks in chain brackets in this case was due to the production process, because the wrinkling of the material appeared during hot bending.

Sujata et al [4] in their study using visual examination found a shallow crack on the surface of the chain link. Under stereo-binocular microscope, the authors found that the fracture surface showed coarse crystalline features. The sample containing the crack was cut, mounted, metallographically prepared and observed under an optical microscope. The visual examination revealed a crack-like surface defect and the optical microgragh 
showed oxide entrapment in the material near the surface. It was observed that that the crack-like defect is not perpendicular to the surface. In between the cracks surfaces, the authors used Energy Dispersive X-Ray (EDX) analysis in SEM for investigation and found that the non-metallic inclusions are mainly iron oxide. He concludes that the conveyor chain links have failed due to presence of defects that is manufacturing-in defect. The investigation also showed that surface defects were present in the billet itself. So he recommends that the billet be properly dressed and the surface defects are removed prior to the forging operations

Conwell and Johnson [5] investigated experimentally, the dynamic behavior of roller chain drives. A strain gage mounted on a link side plate was used to determine chain tension during normal operation over a wide range of linear chain speeds and preloads. The test machine also included specially instrumented idler sprocket that allowed the measurement of the horizontal and vertical components of the bearing reaction force. The roller-sprocket impact force was then computed by an experimental transfer function approach facilitated by a Bruel Kjaer 2032 dual channel spectrum analyzer. It was observed that the tension in a chain link increases rapidly as the link exits the driven sprocket. The increase in tension occured over less than two sprocket teeth from loose side to tight side. The tension in the chain link then decreased very rapidly as the link entered the drive sprocket. The decrease from tight side to average loose side tension occured over less than two sprocket teeth. The impact force tended to increase as chain tension and speed increased.

Khaled et. al [6] investigated failure of Grade-80 alloy steel towing chain links. By using optical metallography and Scanning Electron Microscopy (SEM) analysis, the fatigue failure was found to result from the generated cracks at outer circumference of the weld. The fatigue crack propagation was evident through progressive marks and striations as shown in Figure 3. Points 1, 2, 3 and 4 on the figure represent fatigue origin, fatigue progressive marks and final rupture, void formation and step-like brittle rupture respectively

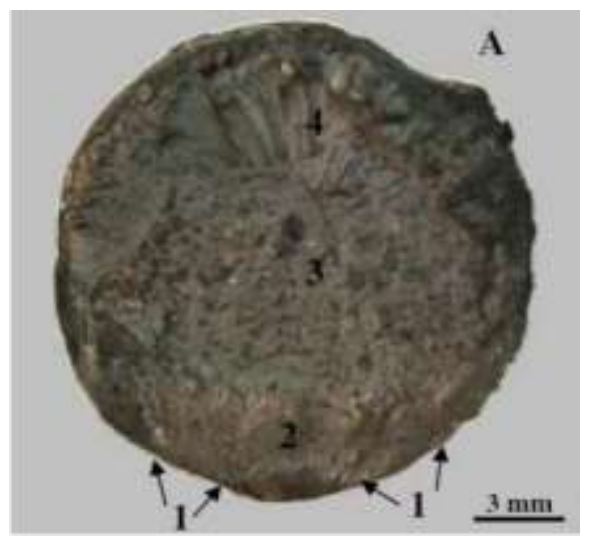

Fig. 3 Fatigue Crack Propagation

The authors concluded that, the evidence of lack of some key alloying elements, welding defects and improper post weld heat treatments of the chain links led to the failures.

Jagtap, et. al [7] studied analytically, experimentally and numerically the behavior of a roller conveyor chain strip under tensile loading. Comparison was made between the three methods and the results showed that they are within $+/-10$ percent of the calculated working stress. The fatigue cracks initially nucleated at the external cracks of the link, and later propagated to the inside of the links until sudden fracture occurred. It was concluded that a roller chain drive may be subjected to tensile loads, thus it must have high tensile strength to withstand the wide range of tensile loads that may be imposed on it.

Singh et.al [8] studied the failure of bridle chain used for hoisting in the mines. Laboratory examination proved that the defect is a mechanically induced one. Visual and stereo-binocular observations revealed surface defects in samples. It was observed that it was not safe to strain the chain to beyond the elastic limit of the material. It was concluded that the cause of failure was as a result of inherited defects in the material and that the chain can fail mechanically by overloading, fatigue and wear.

Bonjak et. al [9] carried out failure analysis on a Stacker Crawler Chain Link. The goal of the study was to diagnose the cause of chain link breakdown occurrence. Working stresses in the chain link were calculated by applying FEM. Experimental investigations were also carried out; chemical composition analysis, tensile properties, the impact toughness and the macro and micro-hardness. Metallographic examinations were also conducted. Based on the results of the numerical-experimental analysis, it was concluded that chain link breakdown is predominantly caused by (a) substantial deviation of the mechanical properties of the material with respect to those prescribed by the standard and (b) the existence of macro and micro cracks in the material structure 


\section{Methodology}

Fractography and metallurgical analysis were employed in this study to analyze the cause of failure on a failed conveyor link of a central bucket elevator. The methodologies to be adopted in this study to establish the cause of failure are as follows;

\subsection{Preliminary Examination}

Firstly, preliminary examination was done visually after a failed sample had been collected. Visual examination was then aided by using stereo microscope to see more clearly the fracture surfaces and surface defects. This step is to examine fracture surfaces and to identify whether the fracture is ductile or brittle. Chevron marks always appear at a fractured surface as a result of the fracture process. Chevron marks are very helpful because they can point to the crack origin. The bucket elevator was studied with special attention paid to the operating conditions such as temperature. Other parameters such as speed, capacity, weight of chain link etc. will be taken. The fractured samples was taken from East African Portland Cement Company (EAPC) for analysis. Preliminary examination was done on fractured samples with stereo microscope to magnify the fractured surface for analysis. Before fractured sample was examined using the stereo microscope, it was first sectioned at $12 \mathrm{~mm}$ from the fractured surface with hacksaw (low speed cutter) so as not to alter the micro structure. The sectioned sample was then mounted on a stereo microscope to observe the grains on the surfaces so as to deduce the type of fracture. Also the mechanism of failure was deduced by tracing the chevron mark on the surface

\subsection{Chemical Analysis}

Chemical analysis at the fractured surface was done to determine the chemical composition. The sectioned sample at the fractured surface used for visual examination was taken for chemical Analysis with mass spectrometer at Numerical Machining Complex limited in Nairobi. Chemical analysis provided information regarding any deviation from the standard specifications, compositional inhomogeneities, impurities, inclusions and segregations. Identification of these harmful constituents is very important in failure analysis because impurities are known to cause embrittlement in metals and segregation of constituent elements sometimes provides an easy path for crack propagation. The sample was cleaned with emery cloth to remove any contamination before it was mounted on the mass spectrometer. Three sparks was introduced unto the sample at three different points to determine the composition of the material. Average of the three sparks at the three different points was found to obtain the average percentages of each of the constituent's elements. Fig 4 shows mass spectrometer with the sample mounted on it.

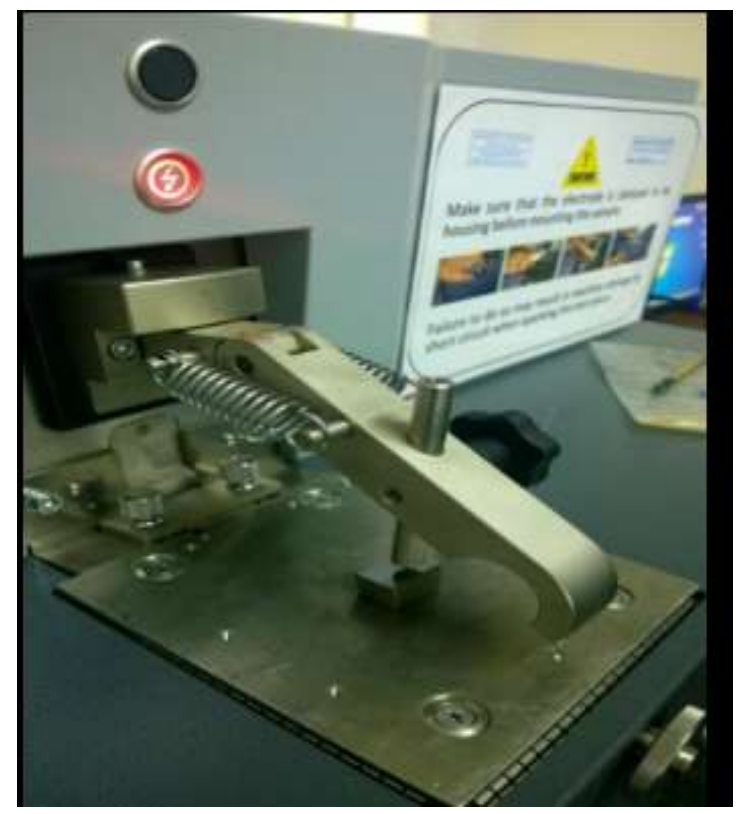

Fig.4 Chemical analysis using mass spectrometer

\subsection{Polishing of Samples}

Before SEM and Metallurgical analysis could be done, the samples needed to be firstly polished.

The polishing equipment's used were:

1. Silicon carbide papers 
2. Polishing cloth

3. Polishing deck

4. Dryer

5. Polishing machine

The polishing reagents used were:

1. Methanol

2. Diamond paste

3. Lapping fluid

4. Nitric acid

The following polishing procedures was adopted to obtain the polished samples as follows;

- The already sectioned samples were grinded on four (4) silicon carbide papers of grade 220, 320, 400 and 600 .

- After grinding is was completed, the specimen was washed with water flashed with methanol and dried with a drier before polishing on a polishing machine.

- Polishing was done using 6 and 4 micron diamond paste. The diamond paste was put on the polishing cloth. Lapping fluid was put on the polishing cloth with the diamond paste.

- After the 4 micron diamond paste was used to finish the samples, its then washes with water, flashed with methanol and then dried with a drier.

\subsection{Preliminary Examination}

\section{Results and discussions}

The preliminary examination done on the factory site revealed indentations on the chain links which is as a result of the sprocket impacting on the chain link as shown in the Fig 5.

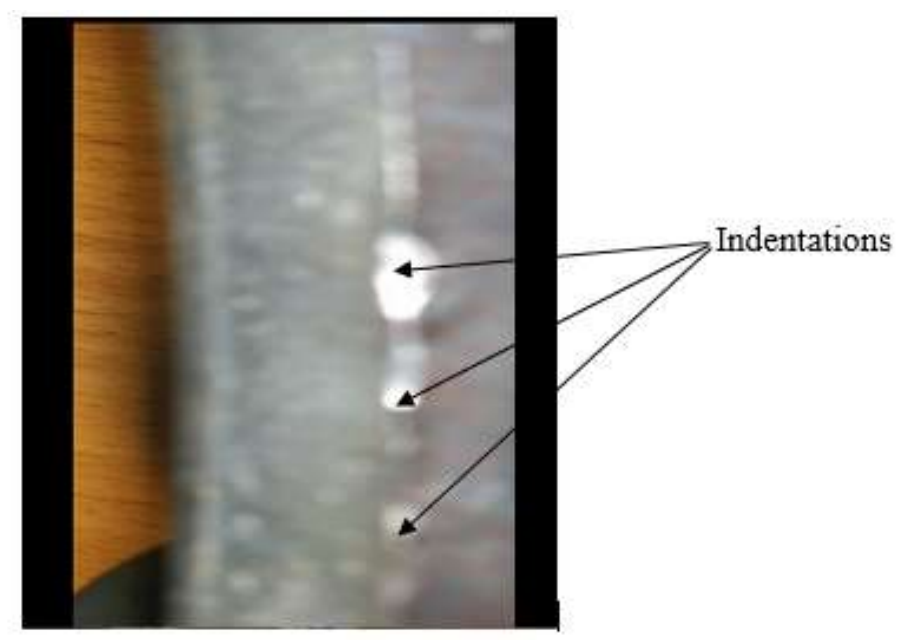

Fig. 5 Indentations on Chain Link

This indicates misalignment and vibrations within the bucket elevator system. Examining the sample under the stereo microscope revealed that the grains at the fractured surface were fine pointing to the fact that it's a brittle fracture. Brittle fracture also occur as a result of induced inclusions at the grain boundaries [12]. Fig 10 and 11 shows that the fracture was induced by inclusions which emphasizes that it's a brittle fracture

Also the mechanism of failure was obtained by tracing the chevron mark on the surface. The fracture started from the core of the fractured surface B of the link and proceeded until it snapped i.e. A $\rightarrow \mathrm{B} \rightarrow \mathrm{C} \rightarrow \mathrm{D}$ $\rightarrow$ E. The mechanism of failure is as shown in the Fig 6. Fig 6 (a) shows that the crack begun at point A and progressed gradually to the surface at point B. Since the chain link is under tensile loading and the crack had already been initiated at $\mathrm{A}$ and grown to $\mathrm{B}$, it then progressed from $\mathrm{C}$ to $\mathrm{D}$ and finally fractured at $\mathrm{E}$. The initiation and growth of the crack from A to B is as shown in Fig 6 (b) 


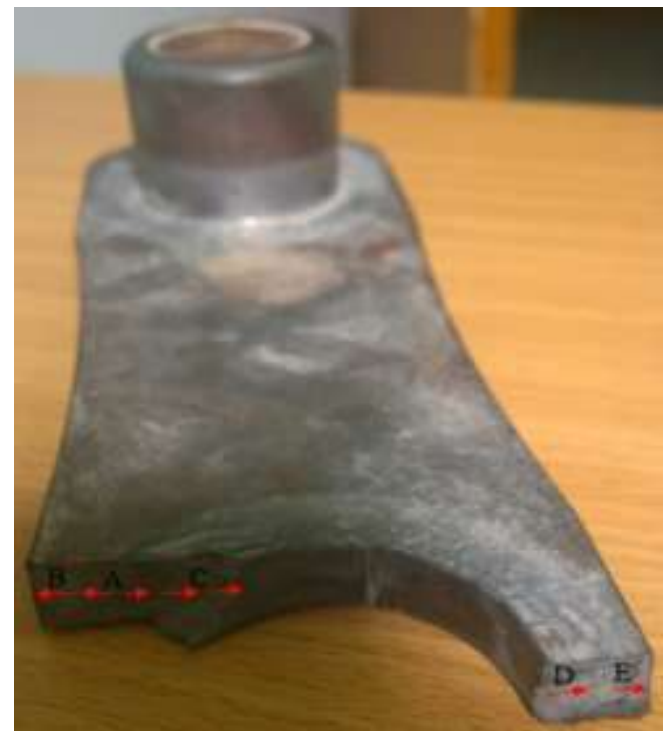

(a)

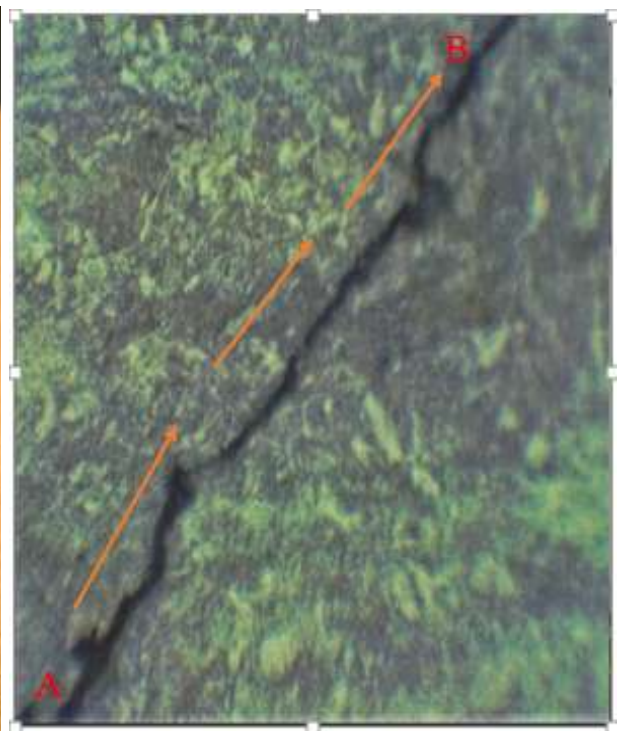

(b)

Fig. 6 Fracture Mechanism

\section{A. Chemical Analysis}

The chemical analysis was performed on five samples and the following results were yielded as shown in Table 2

Table 2: Chemical analysis results

\begin{tabular}{|l|l|l|l|l|l|l|l|l|}
\hline Sample & Element & $\mathrm{C}$ & $\mathrm{Si}$ & $\mathrm{S}$ & $\mathrm{P}$ & $\mathrm{Mn}$ & $\mathrm{Cr}$ & $\mathrm{Mo}$ \\
\hline 1 & Content (\%) & 0.131 & 0.345 & 0.0002 & 0.000510 & 1.49 & 1.47 & 0.174 \\
\hline 2 & Content (\%) & 0.133 & 0.361 & 0.0003 & 0.000581 & 1.51 & 1.50 & 0.183 \\
\hline 3 & Content (\%) & 0.135 & 0.372 & 0.00042 & 0.000652 & 1.51 & 1.54 & 0,180 \\
\hline 4 & Content (\%) & 0.202 & 0.429 & 0.00015 & 0.000552 & 1.48 & 1.60 & 0.179 \\
\hline 5 & Content (\%) & 0.129 & 0.331 & 0.00035 & 0.000592 & 1.49 & 1.61 & 0.175 \\
\hline & Standard (\%) & $0.27-0.34$ & Max 0.6 & Max 0.02 & Max 0.025 & Max 0.5-1.7 & Max 1.3-1.7 & Max 0.15-0.5 \\
\hline
\end{tabular}

The two elements that are detrimental to steel are Sulphur and phosphorus. Sulphur promotes internal segregation in the steel matrix. Both Sulphur and phosphorus act to reduce the ductility weldability of the material. They must therefore not be more than 0.020 for sulphur and 0.025 for phosphorus. From the chemical analysis they are all in range. Carbon plays an important role in the hardness and ductility of steels. The higher the carbon content the harder the steel but the ductility reduces and vice versa. For this reason the carbon content for steels is limited to 0.15- 0.3. From the chemical analysis, the carbon content is below 0.15 and therefore does not meet the required standards.

\subsection{Optical Microscope Examination}

After polishing of the samples was completed, it was viewed under light microscope both etched and unetched. The unetched polished sample showed a lot of blow holes which is a casting defect as shown in figure 7 (a) and oxide formation within the material as shown in figure 7 (b) within the material as shown in the figure below;

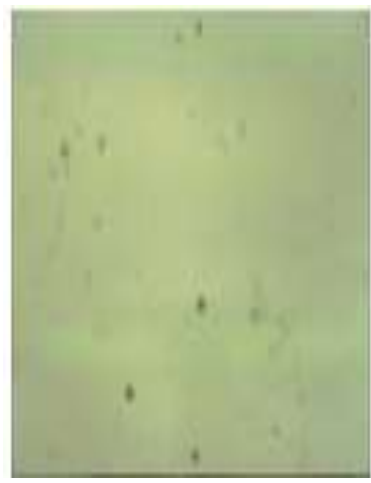

(a)

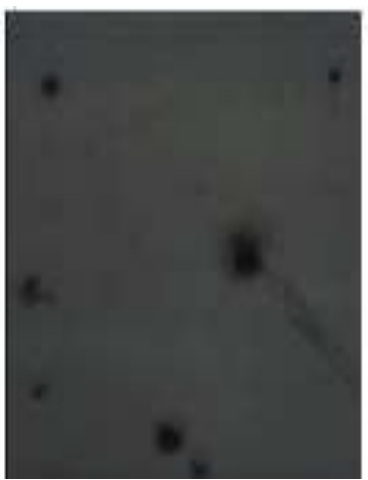

(b)

Fig. 7 Blow holes and oxide formation 
The type of oxide will be determined by using SEM equipped with Energy Dispersive X-Ray (EDX) to verify if the oxide is detrimental to the steel material or not.

The polished samples was then etched in an etchant made of $98 \%$ methanol and $2 \%$ nitric acid (Nital). The microstructure was observed as in the figure below;

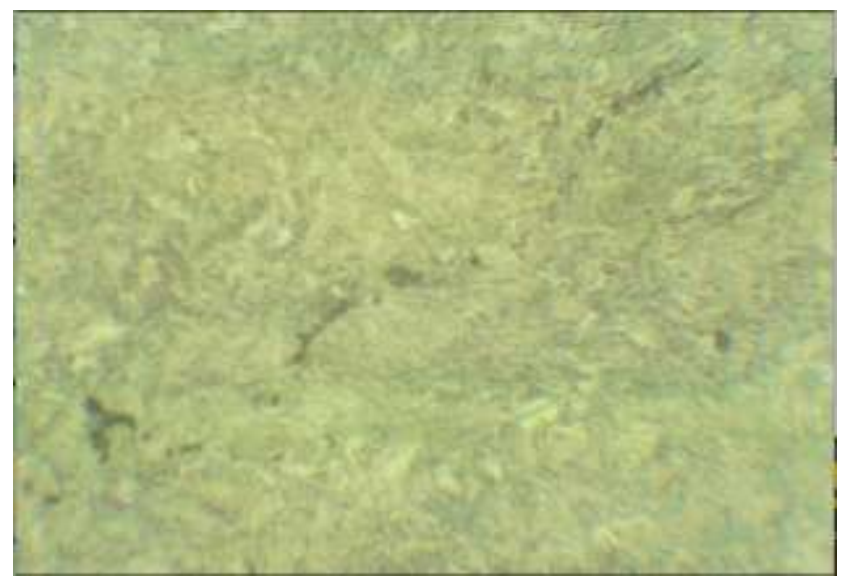

Fig. 8 Microstructure

The micro structure is martensitic which accounts for high strength and wear resistance because of its application. From the micro structure it can be deduced that the material has undergone heat treatment. The micro structure showed a lot of existing cracks within the material introduced during manufacturing as shown in the fig 9 . These cracks then propagated during loading leading to the eventual fracture of the material.
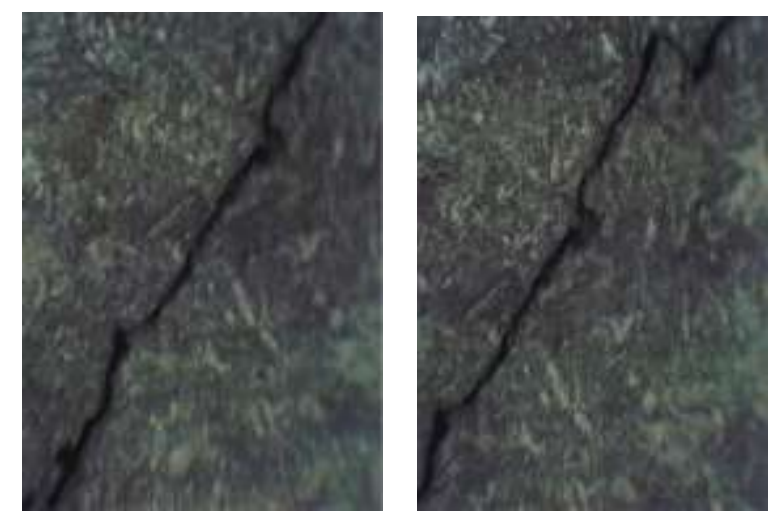

Fig. 9 Cracks within material

The crack began from inclusion $\mathrm{A}$ and progressed until it reached inclusion B. From inclusion B, two cracks emerged, one in the direction of $\mathrm{X}$ and the other in the direction of $\mathrm{Y}$ as shown in the figure 10.

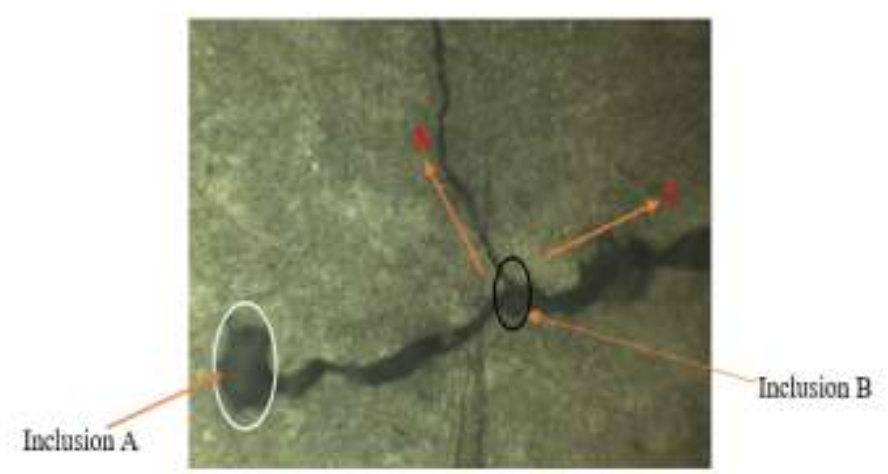

Fig. 10 Crack initiation 


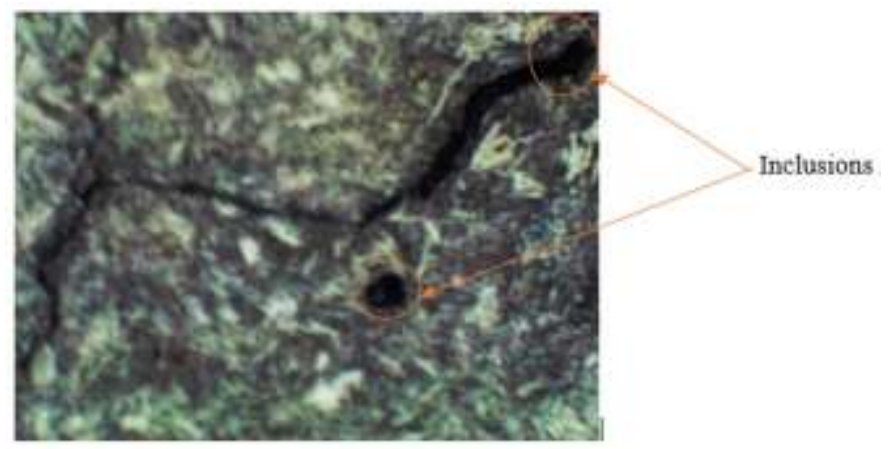

Fig 11 Crack Initiation

These cracks had initiated from an inclusion as shown in figure 10 and 11 under magnification of 500x. Inclusions are produced when the quantity of metal added is greater than necessary to kill steel. The remaining metals combines with oxygen to form inclusions [10]. The metal usually used in killing steels is aluminum. Inclusion are known to have low formability and during loading they produce cracks in the steel. Since inclusions cannot be eliminated completely from steels it is imperative to modify them in terms of chemical composition to minimize their harmful effect with calcium so they become harmless [11]. Calcium (modifier) addition is therefore the solution to eliminating these inclusions by chemically reacting with the inclusion to form calcium aluminate. Calcium aluminate improves machinability, toughness and surface quality rather than being a crack initiation point.

\section{Conclusion}

Failed samples of bucket elevator chain link was collected from East African Portland Cement (EAPC) for analysis. Preliminary examination revealed that the chain link had undergone brittle fracture and the fracture started from the core A and proceeded until it fractured completely. Chemical analysis revealed that the carbon content was not within the prescribed standards. Metallurgical analysis revealed that the crack had initiated from inclusions which then propagated until eventual fracture occurred.

\section{References}

[1]. S. Patel, S. Patel, and J. Patel, “A Review on Design and Analysis of Bucket Elevator,"International Journal of Engineering Research and Applications (IJERA), vol. 2, no. 5, pp. 18-22, 2012.

[2]. N. Yashaswini, B. Raju, and A. Purushottham, "Design and Optimization of Bucket Elevator through Finite Element Analysis," IPASJ International Journal of Mechanical Engineering (IIJME), vol. 2, no. 9, pp. 73-78, 2014.

[3]. D. Momcilovic, N. Hut, L. Milovic, and I. Atanasovska, "Failure Analysis of Chain Bracket," New Trends in Fatigue and Fracture 2011, vol. 11, no. 1, pp. 123-126, 2011.

[4]. M. Sujata, M. A. Venkataswamy, M. A. Parameswara, and S. K. Bhaumik, "Failure Analysis of Conveyor Chain Links," Engineering Failure Analysis, vol. 13, no. 6, pp. 914-924, 2006.

[5]. J. C. Conwell and G. E. Johnson, "Experimental Investigation of Link Tension and Roller-Sprocket Impact in Roller Chain Drives," Mechanism and Machine theory, vol. 31, no. 4, pp. 533-544, 1996.

[6]. N. I. B. Haris, Failure Analysis of Conveyor Chain Links: A Case Study at Top Glove SDN. BHD. PhD thesis, Universiti Tun Hussein Onn Malaysia, 2013.

[7]. Jagtap M. D., G. B. D., and P. P. M., "Study of Roller Conveyor Chain Strip under Tensile Loading," International Journal Of Modern Engineering Research (IJMER), vol. 4, no. 5, pp. 61-66, 2014.

[8]. U. Singh, M. K. Singh, and M. Singh, "Failure Analysis of Bridle Chain Used for Hoisting in Mines," vol. 4, no. April, pp. 38-45, 2013.

[9]. S. M. Bo`snjak, M. A. Arsic, N. D. Zrnic, Z. D. Odanovic, and M. D. Dordevic, "Failure Analysis of the Stacker Crawler Chain Link," Procedia Engineering, vol. 10, pp. 2244-2249, 2011.

[10]. V. Thapliyal, A. Kumar, D. G. C. Robertson, and J. D. Smith, "Transient inclusion formation and evolution in silicon killed steels," Journal of ironmaking and steelmaking, vol. 42, no. 5, pp. 382-394, 2015.

[11]. J. Prez, Inclusion control model in the ladle metallurgy furnace. PhD thesis, Mc Master University, 2012.

[12]. J. Pokluda and P. Sandera, "Brittle and ductile fracture," Micromechanisms of Fracture and Fatigue: In a Multiscale Context, vol. 1, pp. 69-123. 